\title{
Childhood-onset keratosis lichenoides chronica: a case report
}

\author{
Soheila Nasiri ${ }^{1}$, Azadeh Rakhshan ${ }^{2}$, Khatere Zahedi ${ }^{1}$, Sahar Dadkhahfar ${ }^{3}$, and Mehdi \\ Gheisari $^{4}$ \\ ${ }^{1}$ Skin Research Center Shahid Beheshti University of Medical Sciences \\ ${ }^{2}$ Shahid Beheshti Univ Med Sci \\ ${ }^{3}$ Affiliation not available \\ ${ }^{4}$ Shahid Beheshti University of Medical Sciences
}

October 21, 2020

\begin{abstract}
Keratosis lichenoides chronica (KLC) is a rare dermatosis which represents different clinical characteristics between adult- and pediatric-onset cases. We described a childhood case of KLC with features typical for adult onset disease. Acitretin led to partial improvement of her skin (but not mucosal) lesions.
\end{abstract}

\section{Key Clinical Message}

Keratosis lichenoides chronica (KLC) is a rare dermatosis which is manifested as combination of lichenoid keratotic papules with a characteristic linear/reticular arrangement and an erythematous facial eruption.

\section{Introduction}

Keratosis lichenoides chronica (KLC) is a rare dermatosis with a chronic progressive course. Characteristically KLC presents as violaceous, keratotic, lichenoid papules with a linear or reticulated arrangement with symmetrical distribution on the extremities and the trunk (1). Another prominent feature is a seborrhoeic dermatitis- or a rosacea-like eruption on the face. Additionally, oral manifestations such as recurrent aphtha, large chronic ulcers or erythrokeratotic papules occur in $50 \%$ of patients.

Although KLC manifests more commonly in adults (20-40 years), there are some reports of the disease appearing in childhood $(1,2)$. It has been suggested that the disease represents different clinical characteristics between adult- and pediatric-onset cases(3). We describe a patient with the manifestations of adult-onset KLC beginning from infancy.

\section{Case Report}

A 20-year-old female was referred to our clinic with generalized reticular pruritic erythematous-violaceous eruption. The lesions had appeared during the first year of life on her chest and gradually progressed to the other parts. Repeated courses of topical corticosteroids had been administered without any significant improvement. Her past medical and family history was unremarkable. Examination revealed keratotic violaceous papules arranged in a reticular pattern with symmetrical distribution over the extremities and trunk (Figure 1). The lesions were more confluent on the lateral trunk, breasts, buttocks, and extremities. The individual lesions were erythematous verrucous papules covered by a hyperkeratotic plug that could be removed with difficulty.

There was an erythematous rosacea-like eruption on her face. The neck was involved circumferentially and the scalp was scaly but scalp hair, eyebrows, and eyelashes had a normal thickness and density. The nails 
were dystrophic and greatly thickened and there were many keratotic papules on the palmoplantar surfaces. She had tender erosions on her tongue along with soreness sensation in her oral mucosa. Examination of the other mucosal sites including genital mucosa and conjunctiva was insignificant. There were no signs of internal organ involvement or lymphadenopathy.

Routine laboratory data including complete blood count, ESR, CRP, liver, and renal function tests were normal.

We performed skin biopsy with differential diagnoses of KLC, psoriasis, hypertrophic lichen planus, and pityriasis rubra pilaris. Histologic examination of the skin specimen revealed variable epidermal thickening and areas of acanthosis and atrophy, hyperkeratosis, focal parakeratosis with remnants of neutrophils, lichenoid interface reaction with band-like sub-epidermal infiltration of lymphocytes and a few plasma cells which were compatible with keratosis lichenoides chronica (Figure 2).

The diagnosis of KLC was considered based on typical clinical and histopathologic findings and the patient was started in acitretin $25 \mathrm{mg} /$ daily. This led to gradual improvement of the skin lesions with prominent papular flattening and erythema reduction 3 months after treatment commenced but her oral lesions did not demonstrate any significant improvement.

\section{Discussion}

KLC is considered by some to be a variant of lichen planus while others believe that it has a distinct clinical and histological picture(4). The combination of lichenoid keratotic papules with a characteristic linear/reticular arrangement with an erythematous facial eruption refers to the clinical diagnosis of KLC. The histologic features of KLC are variable and nonspecific; however, the presence of parakeratosis and heavier infiltration than what usually seen in lichen planus may help in differentiation(4).

Although children are occasionally affected, the majority of reported KLC cases are adults. In 2007 RuizMaldonado et al. studied 14 cases of pediatric-onset KLC and compared them with adult-onset KLC (3). They proposed that some features of KLC might characterize pediatric-onset KLC including an early or congenital onset, a positive family history with a probable autosomal recessive inheritance, a greater proportion of male to female, an initial location of lesions on the face with erythematous-purpuric macules, forehead, eyebrow, and eyelash alopecia, the higher frequency of pruritus, and a much lower frequency of mucosal involvement and systemic abnormalities (3). Our patient began to develop skin lesions during the first year of her life making her a pediatric-onset KLC but she presented with features more consistent with those of adult-onset KLC. First, the lesions had appeared on the chest instead of the face. Indeed, her facial rosacea-like lesions were a recent phenomenon. Secondly, extensive oral erosions and prominent nail involvement in our case are other dermatological alterations seen in $50 \%$ and $30 \%$ of adult-onset patients respectively (3) but occurring infrequently in children. Negative family history and lack of alopecia are also in favor of adult-onset disease.

The other less common features of KLC are summarized in Table 1.

KLC has a chronic course with a gradual progression and most cases have failed to show a favorable response to any treatment with a mean follow-up time of 14 years in adults(3). Several anecdotal reports have shown the efficacy of ultraviolet A- and ultraviolet B-light phototherapies, natural light, photochemotherapy, and oral retinoids (acitretin or isotretinoin) plus phototherapy.(3) In our case, the patient showed a partial response to treatment with acitretin but phototherapy was not feasible due to the COVID-19 outbreak. Based on the current data systemic corticosteroids, antimalarial agents, sulfones, gold, and cyclosporine are proven to be ineffective in the treatment of $\mathrm{KLC}(4)$.

\section{Conclusion}

This case is a pediatric-onset KLC patient that was remained undiagnosed for several years. This patient had a partial response to oral acitretin highlighting the importance of dermatologists' vigilance about this condition and its proper management if possible. 


\section{Acknowledgement: None}

Conflict of Interest:None

Funding sources: None

Author contributions

Soheila Nasiri: Clinical evaluation and management of the patient, writing the manuscript and supervision of the project.Azadeh Rakhshan: Histopathology evaluation of the patient, writing the related part in the manuscript. Khatere Zahedi: Data gathering, writing the manuscript in consultation with Mehdi Gheisari and Sahar Dadkhahfar. Sahar Dadkhahfar and Mehdi Gheisari : Taking photos and writing and editing of the final draft of the manuscript.

\section{Ethical considerations}

The patient in this manuscript has given written informed consent to the publication of her case details.

\section{References}

1. Patrizi A, Neri I, Passarini B, Varotti C. Keratosis lichenoides chronica: a pediatric case. Dermatology. 1995;191(3):264-7.

2. Masouyé I, Saurat J-H. Keratosis lichenoides chronica: the centenary of another Kaposi's disease. Dermatology. 1995;191(3):188-92.

3. Ruiz-Maldonado R, Duran-McKinster C, Orozco-Covarrubias L, Saez-de-Ocariz M, Palacios-Lopez C. Keratosis lichenoides chronica in pediatric patients: A different disease? Journal of the American Academy of Dermatology. 2007;56(2):S1-S5.

4. Konstantinov KN, Søndergaard J, Izuno G, Obreshkova E. Keratosis lichenoides chronica. Journal of the American Academy of Dermatology. 1998;38(2):306-9.

5. Mehregan A, Heath L, Pinkus H. Lichen ruber moniliformis and lichen ruber verrucosus et reticularis of Kaposi. Journal of cutaneous pathology. 1984;11(1):2-11.

6. Baran R, Panizzon R, Goldberg L. The nails in keratosis lichenoides chronica: characteristics and response to treatment. Archives of dermatology. 1984;120(11):1471-4.

7. Margolis MH, Cooper GA, Johnson SA. Keratosis lichenoides chronica. Archives of dermatology. 1972;105(5):739-43.

8. Nijsten T, Mentens G, Lambert J. Vascular variant of keratosis lichenoides chronica associated with hypothyroidism and response to tacalcitol and acitretin. Acta dermato-venereologica. 2002;82(2).

9. Ezzine-Sebai N, Fazaa B, Mokhtar I, Pierard-Franchimont C, Pierard G, Kamoun MR. Keratosis lichenoides chronica: an unusual case. Dermatology. 1996;192(4):416-7.

\section{Figure legends}

Figure 1. Erythematous, scaly, keratotic papules in a reticulated pattern on the (A) buttocks and lower extremity, (B) posterior trunk, (C) and anterior trunk pronounced on the breasts; (D) Dystrophic toe nails; (E) erosive lesions on the tongue.

Figure 2. $(A, B)$ variable epidermal thickening with areas of acanthosis and atrophy, hyperkeratosis, focal parakeratosis with remnants of neutrophils, lichenoid interface reaction with band-like sub-epidermal infiltration of lymphocytes and a few plasma cells. $(\times 4$ objective $)$

Table 1 Additional less frequent features of KLC 
Yellow discoloration of the nails (5)

Yellow discoloration of the nails (5)

Nail dystrophy (6)

Nodular infiltration of the epiglottis, which may cause hoarseness (7)

Vascular lesions (8)

Sclerodactyly (9)

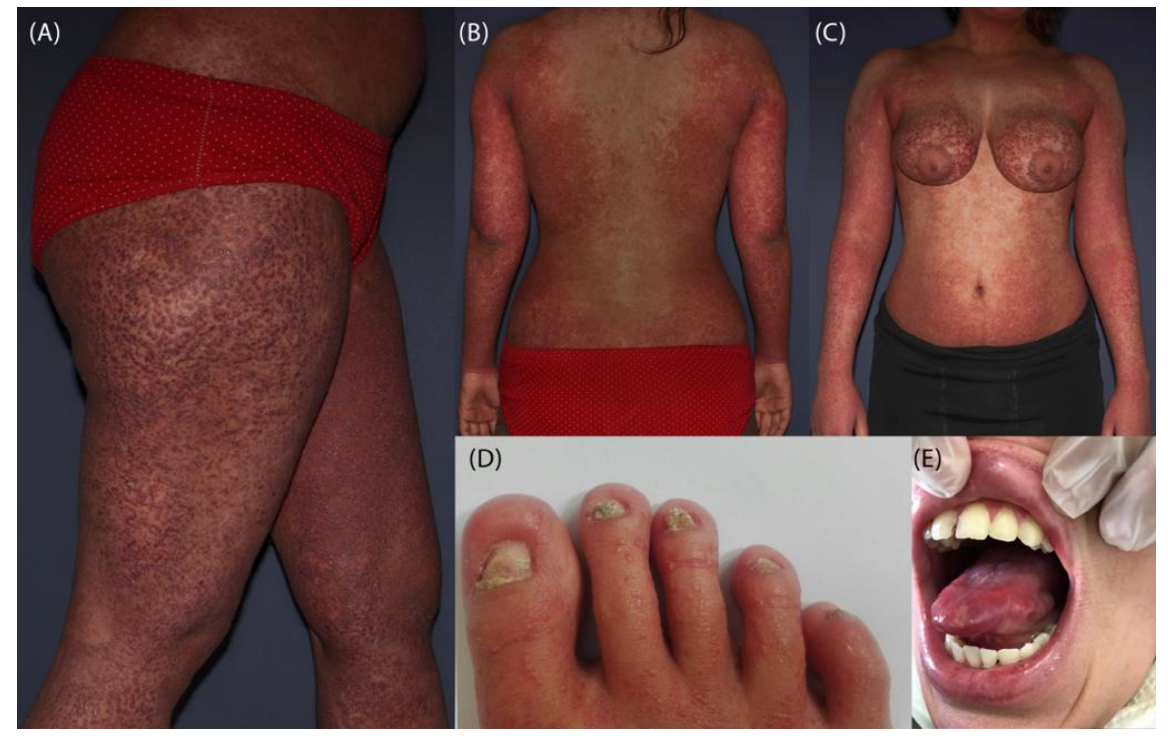




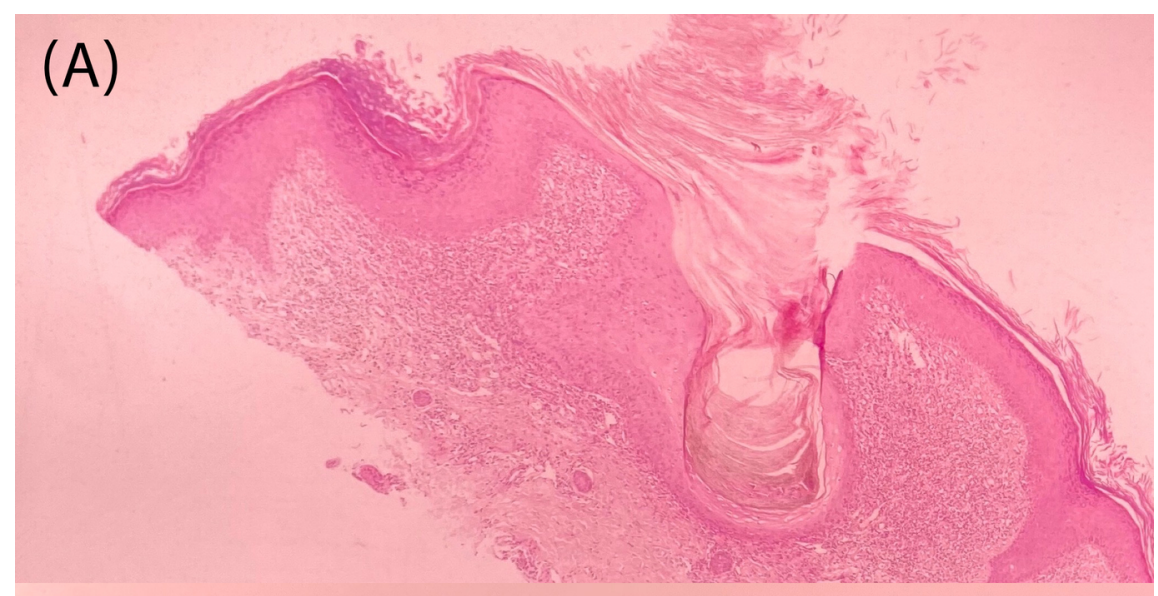

(B)

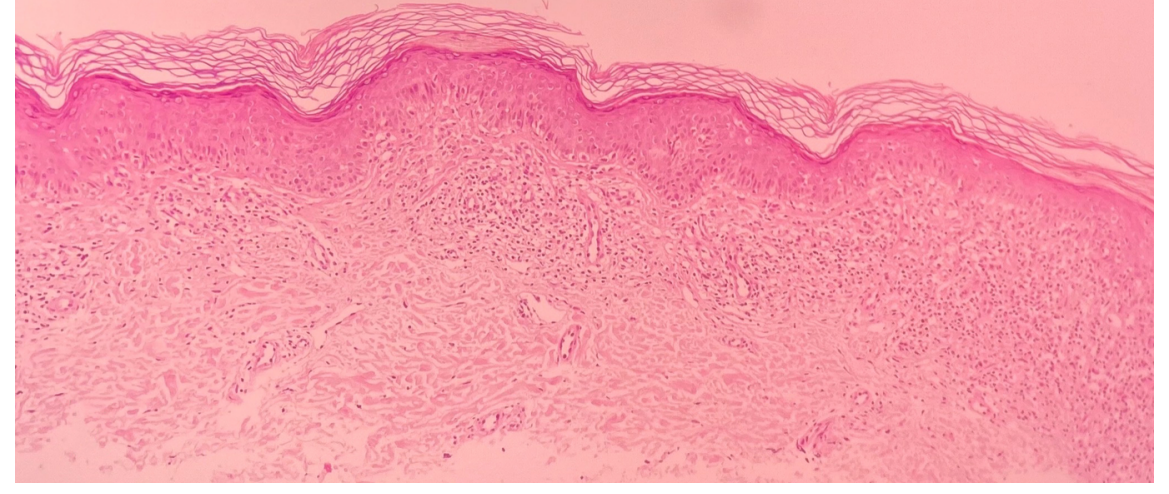

